\title{
Experimental Research and Numerical Simulation on Gas-Liquid Separation Performance at High Gas Void Fraction of Helically Coiled Tube Separator
}

\author{
Yongxue Zhang, ${ }^{1}$ Chan Guo, ${ }^{2}$ Hucan Hou, ${ }^{1}$ and Guomin $\mathrm{Xue}^{3}$ \\ ${ }^{1}$ College of Mechanical and Transportation Engineering, China University of Petroleum, 18 Fuxue Road, Changping, \\ Beijing 102249, China \\ ${ }^{2} 101$ Institution, Ministry of Civil Affairs, Beijing 101601, China \\ ${ }^{3}$ Quality and Safety Environment Ministry of CNPC, Daqing 163000, China
}

Correspondence should be addressed to Yongxue Zhang; zhyx@cup.edu.cn

Received 26 November 2013; Revised 4 May 2014; Accepted 8 May 2014; Published 9 July 2014

Academic Editor: Mostafa Barigou

Copyright (C) 2014 Yongxue Zhang et al. This is an open access article distributed under the Creative Commons Attribution License, which permits unrestricted use, distribution, and reproduction in any medium, provided the original work is properly cited.

\begin{abstract}
The industrial removal process of the light hydrocarbon and water from wet natural gas can be simulated in laboratory with the independently designed helically coiled tube gas-liquid separator. Experiment and numerical simulation are combined to analyze the influences of various inlet velocities and gas void fractions on the gas-liquid separation efficiency and pressure-drop between the inlet and outlet of the helically coiled tube. The results show that, at the inlet velocity of $4 \mathrm{~m} / \mathrm{s}$ to $18 \mathrm{~m} / \mathrm{s}$ and the gas void fraction of $88 \%$ to $97 \%$ for the gas-liquid mixture, the gas-liquid separation efficiency increases at the beginning and then decreases with increasing inlet velocity. Afterwards there is another increasing trend again. The gradient of pressure-drop increases slowly and then fast with the increasing inlet velocity. On the other hand, the gas-liquid separation efficiency first increases with the gas void fraction and then shows a decreasing trend while the pressure-drop keeps falling down with the gas void fraction increasing. Above all the optimal operating parameters of the helically coiled tube separator are inlet velocity of $13 \mathrm{~m} / \mathrm{s}$ and gas void fraction of $93 \%$, and the separation efficiency and pressure-drop are $95.2 \%$ and $0.3 \mathrm{MPa}$, respectively.
\end{abstract}

\section{Introduction}

With the rapid growth of energy demand in the world [1], the resources of offshore oil-gas and desert oil-gas have entered the stage of large-scale development [2-4], and the natural gas which could be directly exploited is mainly wet natural gas containing a small amount of solid particles. Wet natural gas (gas void fraction approximately from 90 to 99\%) always refers to such kind of gas containing a certain amount of water or light hydrocarbon in petroleum and natural gas industry. In order to meet the process requirements of the pipeline transportation and accuracy measurement, it is necessary to carry out the gas-liquid separation for wet natural gas. However, due to the limitation of site conditions and process equipment, a good gas-liquid separator generally has to meet some characteristics, including small size, high separation efficiency, high processing capacity, and easy maintenance. Due to these characteristics, as a type of new separator, the helically coiled tube gas-liquid separator has obtained high interest in both research and industrial application.

The helically coiled tube gas-liquid separator is one of the new types of separators, which contains the functions of centrifugal separation and gravity separation. As shown in Figure 1, the structure of separator includes gas collection chamber, separation chamber, and liquid collection chamber from top to bottom. When the inlet velocity of wet gas is high, gas-liquid separation performance mainly depends on the larger centrifugal driven force of the helically coiled tube. On the other hand, when the wet gas velocity becomes quite low, the gravity separation plays the lead role on gasliquid separation performance making up for the lack of the insufficient centrifugal force. The separation performance of 


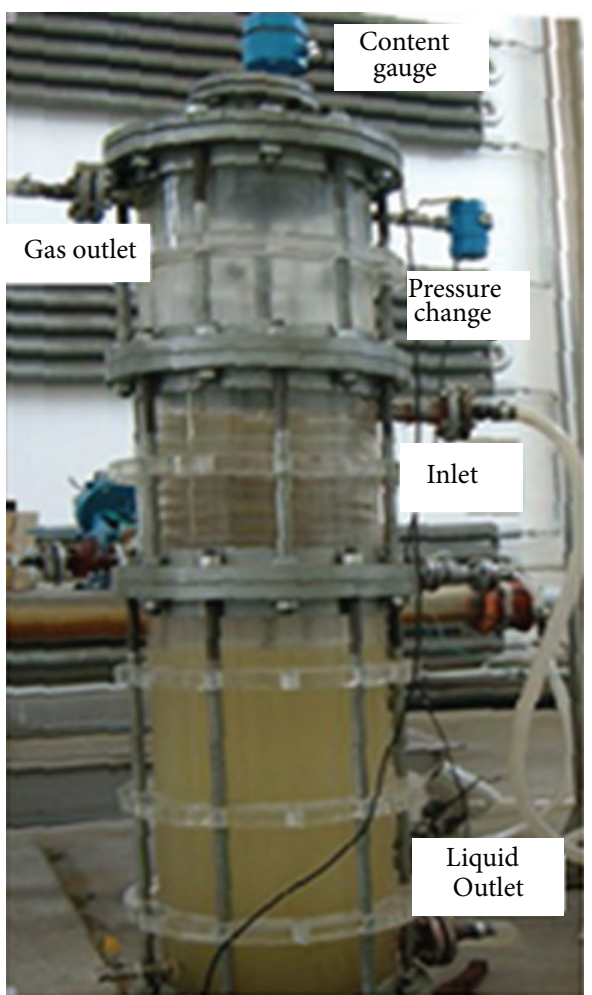

FIgURE 1: The separator structure.

the independently designed helically coiled tube gas-liquid separator has been studied by experiments and numerical simulation. Experimental studies of oil-gas two-phase flow in the horizontal helically coiled pipes with different diameters have been carried out by Guo et al. [5]. Some research works on phase distribution characteristics of solid-gasliquid three-phase flow in helical pipes have been made $[6,7]$. Gong et al. [8] carried out some researches on the numerical simulation with multiphase flow model and the experiments of oil and water separation. They also proposed the best open position on the wall of helical pipe under low flow rate at the inlet. The separation process of oil-water in helically coiled pipes was studied by Li et al. [9-11]. The focus of this paper is the influence of inlet velocity and gas void fraction on the gas-liquid separation efficiency and pressure-drop between the inlet and outlet of the helically coiled tube. The optimal conditions of the separator are studied and analyzed in this paper.

\section{The Experimental Setup}

The whole experimental system is mainly composed of atomization system and separation system $[12,13]$ as shown in Figure 2.

In the atomization system, compressor provides high pressure air and pump transports high pressure liquid water. The high pressure water is atomized into droplet group after nozzle and then injected into the high pressure air. After intensive blend, the gas-liquid mixture enters into the tangential inlet of the separation chamber. Through adjusting the valve, various inlet velocities and gas void fractions of the gas-liquid mixture can be obtained.

As the core of the helically coiled tube gas-liquid separator, the separation system plays the key role in the performance of the separator. Gas-liquid mixture is sent into the entrance of helically coiled tube. After centrifugal separation of the initial circles, air is rapidly discharged into the gas collection chamber along the separation holes inner upside the helical coil tube and water is gathering into the liquid collection chamber. After separation, air is emptied and water is circularly used.

The separator was designed to a cylindrical vertical structure with diameter of $325 \mathrm{~mm}$ and the total height of the separator is $1500 \mathrm{~mm}$ including the gas collection chamber with height of $300 \mathrm{~mm}$, the separation chamber with height of $400 \mathrm{~mm}$, and liquid collection chamber with height of $800 \mathrm{~mm}$. The diameters of inlet and outlet tube of gas and liquid are both $25 \mathrm{~mm}$ [14]. The gas circuit was equipped with a gas flow meter and a pressure-regulating valve. The liquid circuit was equipped with a liquid flow meter and a pressureregulating valve. The separator was equipped with a liquid level-meter.

\section{Experimental Method}

The experimental medium was chosen to be the mixture of water and air, whose physical property is quite similar to the wet natural gas such as low volatility at room temperature. Therefore the mixture can be used to approximately simulate the process of removal of the light hydrocarbon, water, and other liquid phase components from the wet natural gas. At the beginning of the experiment, the valve of the gas circuit before being mixed was kept close, but the inlet valve of the separator, the inlet valve of water circuit, the outlet valve of gas circuit, and the outlet valve of water circuit should be open. The pump was started to establish a certain level of water in the liquid collection chamber. After the stability of liquid circuit system reached a stable state, the air compressor was started and then the valve of gas circuit was opened. By adjusting the vent valve in front of the gas flow meter, a proper air flow can be obtained in the gas circuit. After the stabilization of gas-liquid flow, the separation efficiency can be calculated as follows:

$$
\eta=\frac{Q_{2} t}{Q_{1} t}
$$

where $Q_{1}$ is the reading number of liquid flow meters at entrance of water circuit, $Q_{2}$ is the reading number of liquid flow meters at export of liquid collection chamber, and $t$ is the running time.

\section{Result and Analysis}

During the experiment, the room temperature and barometric pressure are chosen as the experimental temperature and pressure respectively. During the postprocessing calculation, the density and viscosity of water are $1000 \mathrm{~kg} / \mathrm{m}^{3}$ and $5 \times$ $10^{-3} \mathrm{~Pa} \cdot \mathrm{s}$, respectively while those of the air are $1.237 \mathrm{~kg} / \mathrm{m}^{3}$ 


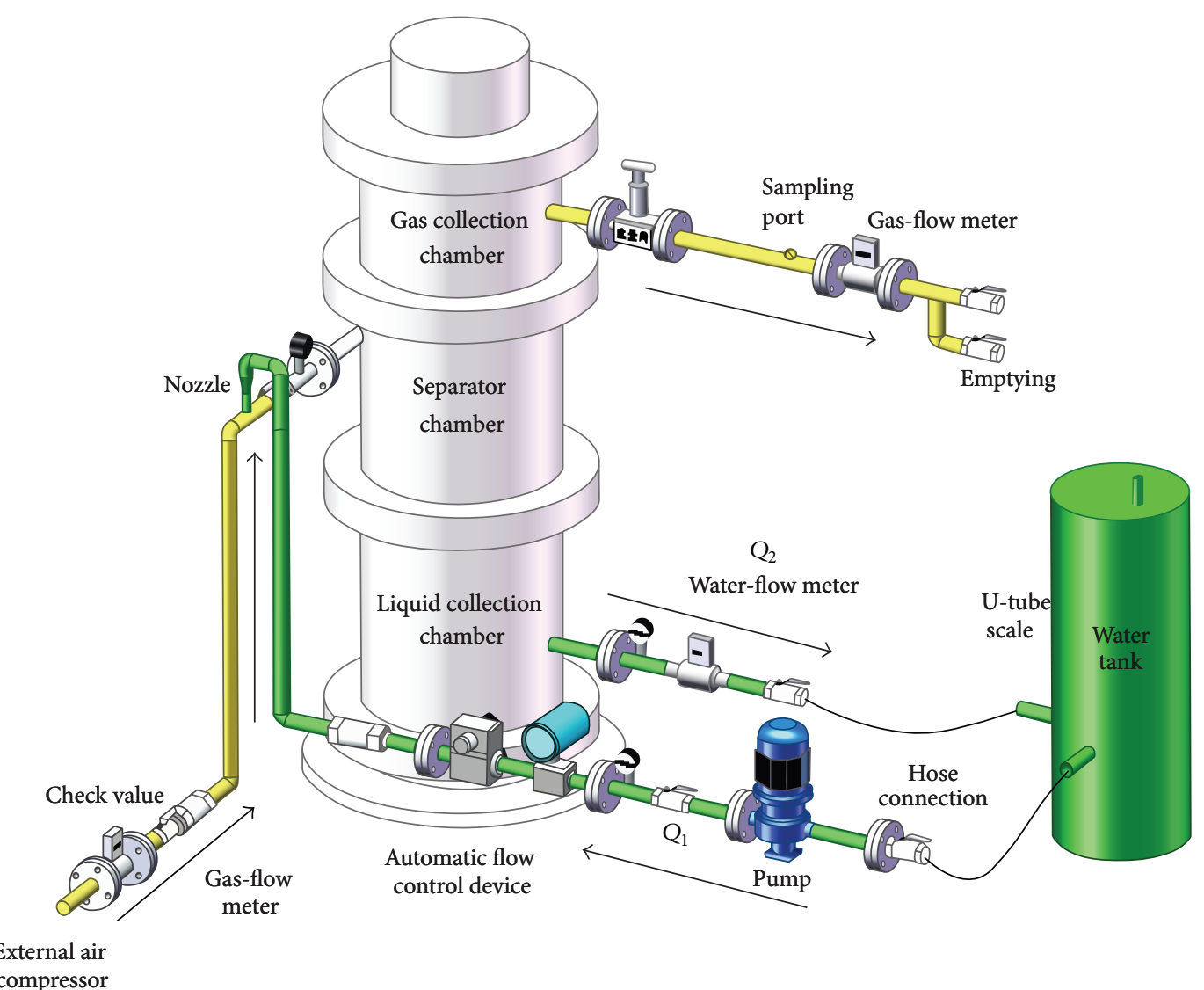

FIGURE 2: The system diagram of the experiment setup.

and $1.822 \times 10^{-5} \mathrm{~Pa} \cdot \mathrm{s}$, respectively. The experiment is mainly concerned with the separation efficiency and pressure-drop which can well reflect the separation performance under different inlet velocities and gas void fractions. The total of 76 conditions can be separated into two parts: one part is at the inlet velocities of $5 \mathrm{~m} / \mathrm{s}, 8 \mathrm{~m} / \mathrm{s}, 10 \mathrm{~m} / \mathrm{s}, 13 \mathrm{~m} / \mathrm{s}, 14 \mathrm{~m} / \mathrm{s}$, $15 \mathrm{~m} / \mathrm{s}, 16 \mathrm{~m} / \mathrm{s}, 17 \mathrm{~m} / \mathrm{s}$, and $18 \mathrm{~m} / \mathrm{s}$ under the gas void fractions of $90 \%, 93 \%, 95 \%$, and $97 \%$ and the other is under the gas void fractions of $88 \%, 89 \%, 90 \%, 91 \%, 92 \%, 93 \%, 94 \%, 95 \%$, $96 \%$, and $97 \%$ at the inlet velocities of $5 \mathrm{~m} / \mathrm{s}, 10 \mathrm{~m} / \mathrm{s}, 13 \mathrm{~m} / \mathrm{s}$, and $18 \mathrm{~m} / \mathrm{s}$.

4.1. The Influence of Inlet Velocity on Separation Performance. Figure 3 represents the influence of the inlet velocity on separation efficiency under different gas void fractions. It can be seen that, at a constant gas void fraction for the gasliquid mixture, the gas-liquid separation efficiency increases with inlet velocity in the range of 5 to $18 \mathrm{~m} / \mathrm{s}$. At an inlet velocity of $13 \mathrm{~m} / \mathrm{s}$ and a gas void fraction of $93 \%$, the separation efficiency reaches the peak value of $95.2 \%$. With the inlet velocity further increasing, the separation efficiency decreases at first and then increases again. The phenomenon is reasonable because the centrifugal force is enhanced with the increasing of inlet velocity leading to better gas-liquid separation efficiency. However, along with the inlet velocity further increasing, large numbers of droplets start to break up

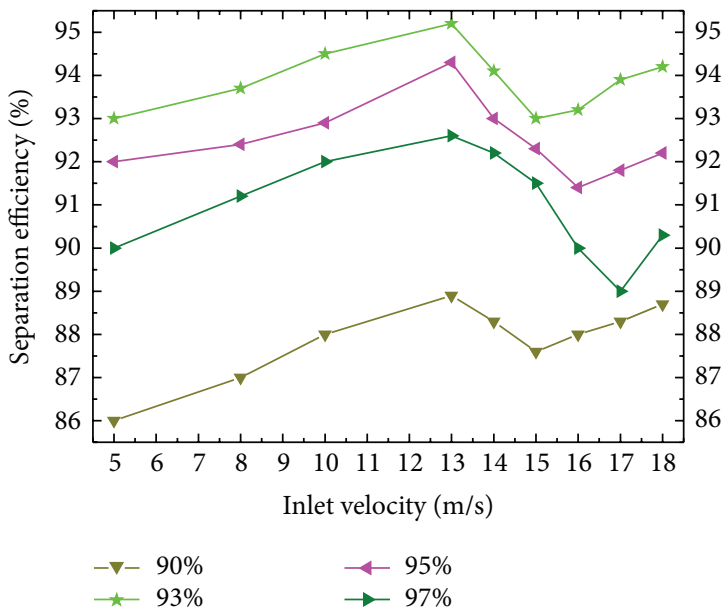

FIGURE 3: The influence of the inlet velocity on separation efficiency under different gas void fractions.

before separation from the gas flow due to the anabatic shear force. Meanwhile the stay time is shortened for the mixture in the helical coil pipe resulting in the increase of pressure-drop and energy consumption.

Figure 4 shows the influence of the inlet velocity on pressure-drop between the inlet and outlet of the helical coil 


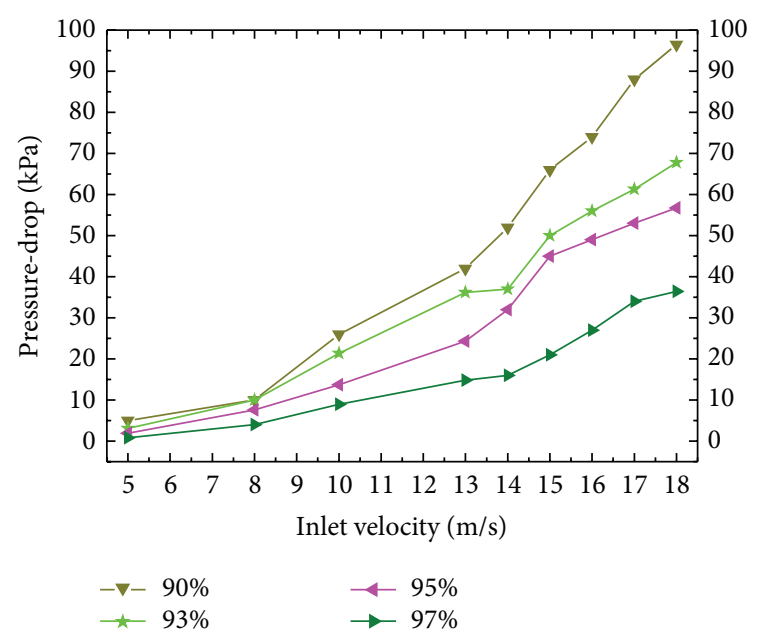

FIGURE 4: The influence of the inlet velocity on pressure-drop under different gas void fractions.

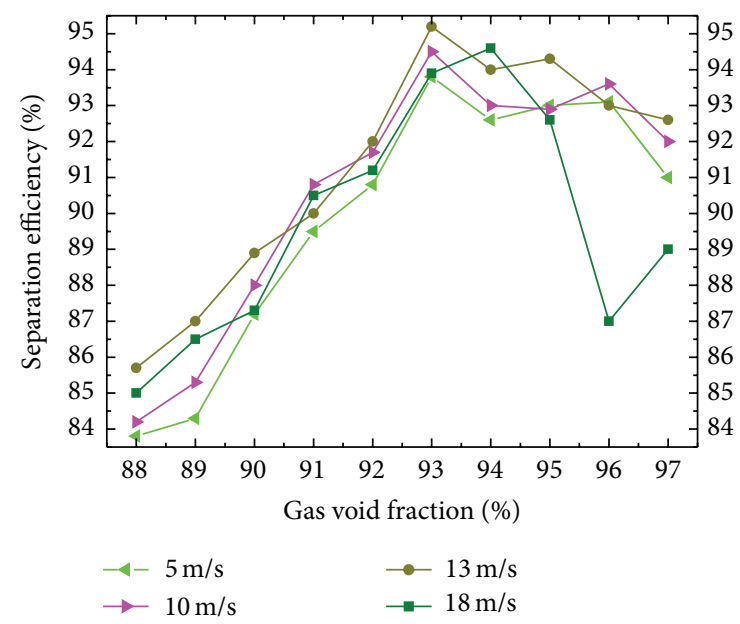

Figure 5: The influence of gas void fraction on the separation efficiency under different inlet velocities.

tube under different gas void fractions. It can be seen that, at a constant gas void fraction for the gas-liquid mixture, the pressure-drop increases with the inlet velocity in the range of 5 to $18 \mathrm{~m} / \mathrm{s}$. At the range of 5 to $14 \mathrm{~m} / \mathrm{s}$ the pressure-drop rises slowly and then it becomes fast at the range of 14 to $18 \mathrm{~m} / \mathrm{s}$, which shows a good manner of quadratic behavior for the transition of pressure drop. The reason can be explained by the fact that the increasing of inlet velocity results in much more flow loss. But with the inlet velocity further increasing, the local loss is rapidly increased resulting in the larger pressure-drop.

\subsection{The Influence of Gas Void Fraction on Separation Perfor-} mance. Figure 5 is the influence of gas void fraction on the separation efficiency under different inlet velocities. It can be seen that, at a constant inlet velocity for the gas-liquid mixture, the gas-liquid separation efficiency first increases and then decreases with gas void fraction in the range of

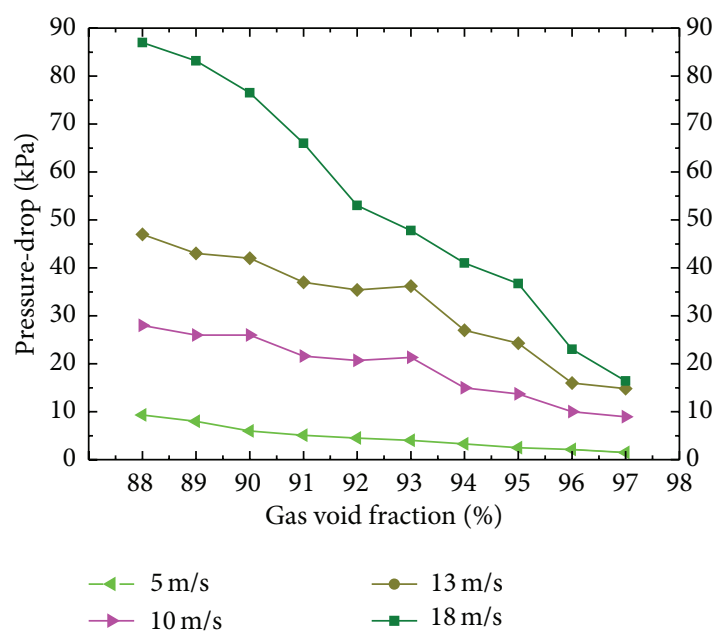

FIGURE 6: The influence of gas void fraction on the pressure-drop under different inlet velocities.

$88 \%$ to $97 \%$. When gas void fraction is at the range of $93 \%$ to $94 \%$, the separation efficiency reaches the peak under different inlet velocities. This is mainly because a bigger gas void fraction can promote the droplet to assemble at the outside of the helical coil tube and forms a continuous gas zone at the inner side, which improves the separation effect. However, with gas void fraction further increasing, the gas phase zone would become intermittent and the flow pattern turns to be mist flow [15]. The flow field becomes unstable and easy-disturbing and consequently the continuous gas blows away the water and makes the water droplet or water mist suspended in the high speed gas stream. So the water droplet is taken away with the discharge of air via holes resulting in bad separation effect.

Figure 6 shows that, at a constant inlet velocity, the pressure-drop decreases with the gas void fraction in the range of $88 \%$ to $97 \%$. This is mainly because, with the gas void fraction increasing, the viscosity of the gas-liquid mixture decreases and the wall shear forces lower resulting in the fall of the pressure-drop.

Taking the above results into comprehensive consideration as well as the separation efficiency and the pressure-drop of the helically coiled tube gas-liquid separator, the separator has an ideal operating condition point, which is the fact that the inlet velocity and the gas void fraction are $13 \mathrm{~m} / \mathrm{s}$ and $93 \%$, respectively, and at this condition the separation efficiency reaches $95.2 \%$ and the pressure-drop is $0.3 \mathrm{MPa}$.

\section{Numerical Simulation}

The gas-liquid separation performance of the helically coiled tube separator with holes is influenced by coupling multiparameters such as inlet velocity, gas void fraction, gas and liquid physical properties, turning radius, pitch, diameter of tube, number of helical turns, and the size of holes [16]. It is impractical for experiment alone to study and optimize these parameters one by one due to huge workload, and more because of the coupling nonlinear of these parameters on the 


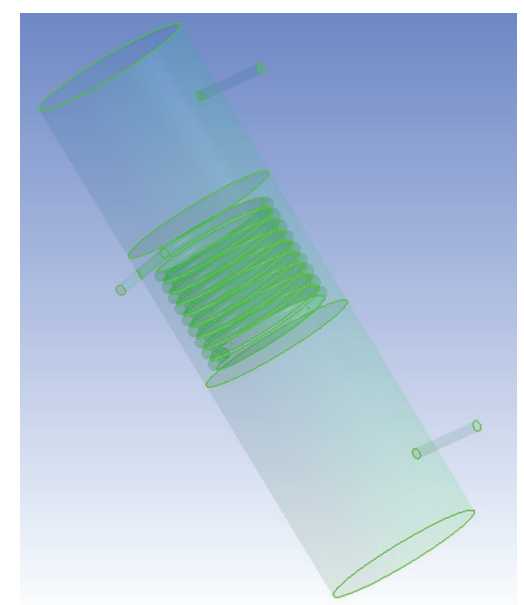

FIgURE 7: The 3D model of the separator.

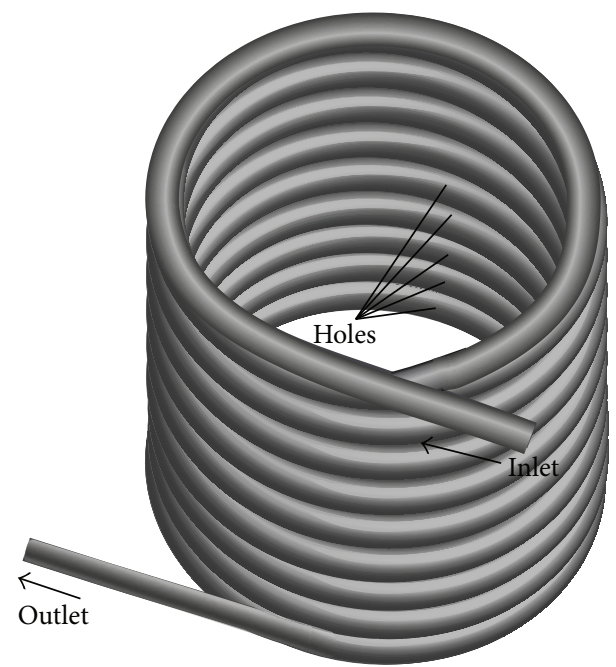

FIgURE 8: The helical coil tube with holes.

separation effect single optimal parameter combination is not really the optimal condition point. Therefore, it is necessary to use the advantages of experimental research and numerical simulation. Through comparative analysis with numerical and experimental results, the use of reliable numerical models to research on gas-liquid separation performance of helically coiled separator is more systematic and in-depth which has a certain guiding significance in the future.

5.1. Meshing and Models. The 3D models of the separator were produced by professional software Ansys DM as shown in Figure 7. In order to reduce the effect of boundary condition on inner flow, the inlet and outlet were extended. The calculation domains were meshed and checked by software Ansys Meshing. Due to the complexity of the helically coiled tube geometry the unstructured tetrahedral and local refinement were used. The quality of the whole mesh was checked less than 0.7 while the number closing to zero represents the best mesh quality in Meshing code, which proved that the grid was good.
Ansys Fluent software was adopted to simulate the inner flow field by assuming that the fluid is steady, viscous, and incompressible [17]. The RNG $k-\varepsilon$ turbulent model and multiphase flow Euler model are applied to close the RANS equations with SIMPLE [18-21] algorithm to solve. It is considered that the calculation is over when the calculation residual is less than 0.001 .

The velocity inlet with a certain gas void fraction and outflow were selected as inlet and outlet boundary conditions assuming that the inlet velocity $u_{\text {in }}$ is distributed uniformly at axis direction. The 19 outflow holes of tube are set at the pressure outlet condition. The turbulent condition at pump inlet can be described by turbulent kinetic $k_{\text {in }}$ and turbulent dissipation rate $\varepsilon_{\text {in }}$ as follows:

$$
\begin{gathered}
u_{\text {in }}=\frac{4 Q}{\left(3.14 \times D_{\text {in }}^{2}\right)}, \\
k_{\text {in }}=0.005 u_{\text {in }}^{2}, \\
\varepsilon_{\text {in }}=\frac{\left(C_{\mu}^{0.75} k_{\text {in }}^{1.5}\right)}{l},
\end{gathered}
$$

where $Q$ is the flow rate of the gas-liquid mixture, $D_{\text {in }}$ is the diameter of the helically coiled tube, $l$ is the turbulent length scale, $l=0.07 D_{\text {in }}$, and $C_{\mu}=0.09$.

As to wall boundary condition, no slip condition is enforced on wall surface and standard wall function is applied to adjacent region.

The helical tube adopts the basic size with gyration radius of $150 \mathrm{~mm}, 9$ laps, and tube diameter of $25 \mathrm{~mm}$ and pitch of $25 \mathrm{~mm}$. The top of helical tube is entrance and the bottom is export. A certain extent in the inlet and outlet reduces the disturbing from reflux on the inner flow field. From the fifth lap the helical coil tube opens the holes uniformly distributed in every $90^{\circ}$ around each lap with the diameter of $3 \mathrm{~mm}$ and location inside the helical tube upper $3^{\circ}$ from the horizontal center. The internal of every two holes with length of a quarter circle of helical pipe without holes is considered as "adjustment segment," facilitating better stratified flow and steady flow as shown in Figure 8.

The grid independence and sensitivity in the simulation have been investigated. Two physical quantities such as efficiency and pressure-drop are chosen as the evaluation parameters for the effect of mesh size on the final solution as shown in Table 1. Then Mesh 3 is employed for the next calculation in order to reduce the calculation loads and guarantee the calculation accuracy. When the effect of grid number on separation performance is less than $1 \%$, the mesh is available. The grid number of the whole structure reaches 4.17 million. Figure 9 gives the meshing of helically coiled tube separator.

\subsection{The Influence of Inlet Velocity and Gas Void Fraction} on Separation Performance of the Helical Tube Gas-Liquid Separator. The influence of inlet velocity on separation performance has been drawn in Figures 10 and 11. It can be seen that, at a constant gas void fraction, the inlet velocity ranging from $5 \mathrm{~m} / \mathrm{s}$ to $13 \mathrm{~m} / \mathrm{s}$ increases the separation efficiency and 

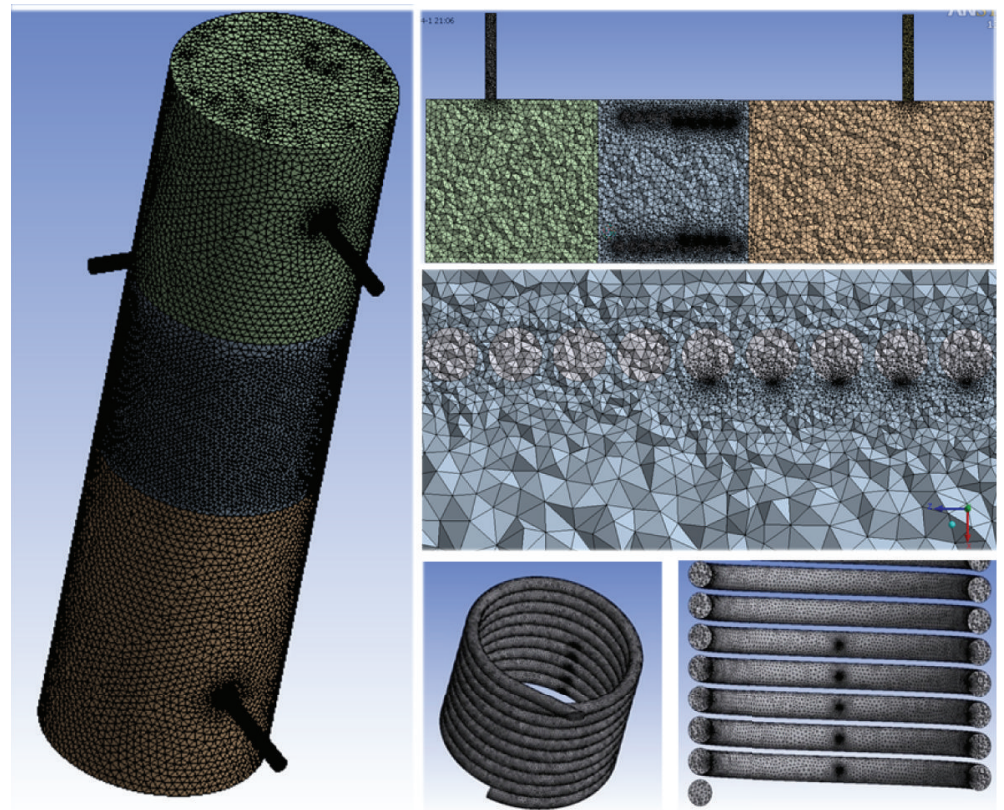

Figure 9: The meshing of helically coiled tube separator.

TABLE 1: Evaluation of the dependency of mesh.

\begin{tabular}{lcccc}
\hline Items & Mesh 1 & Mesh 2 & Mesh 3 & Mesh 4 \\
\hline Total grid & 3489397 & 3951551 & 4176194 & 4527358 \\
\hline$\eta / \eta_{3}$ & 0.998249 & 1.001912 & 1.0 & 1.000697 \\
$\Delta P / \Delta P_{3}$ & 0.998061 & 1.002053 & 1.0 & 1.00767 \\
\hline
\end{tabular}

improves the flow condition. And at $13 \mathrm{~m} / \mathrm{s}$ the separation efficiency reaches the peak value $89.3 \%$ under the gas void fraction of $93 \%$. With the inlet velocity further increasing from $13 \mathrm{~m} / \mathrm{s}$ to $18 \mathrm{~m} / \mathrm{s}$, the separation efficiency decreases at first and then increases slowly again. Meanwhile at the range of 5 to $14 \mathrm{~m} / \mathrm{s}$ the pressure-drop rises slowly and then it becomes fast at the range of 14 to $18 \mathrm{~m} / \mathrm{s}$ with the same regularity to the experiment.

Figures 12 and 13 perform the influence of gas void fraction on separation performance. It can be seen that, at a constant inlet velocity for the gas-liquid mixture, the gasliquid separation efficiency increases with gas void fraction firstly in the range of $90 \%$ to $93 \%$. And at a gas void fraction of $93 \%$ and an inlet velocity of $13 \mathrm{~m} / \mathrm{s}$, the separation efficiency reaches a peak value of $89.3 \%$. When the gas void fraction changes from $93 \%$ to $97 \%$, the separation efficiency shows a downward trend. At the same time, the pressure-drop also decreases with gas void fraction increasing.

\subsection{The Flow Field on Holes Section of the Helically Coiled} Tube Gas-Liquid Separator. Figure 14 shows the flow field and contour of gas void fraction at holes section of the 5 9 helically coiled tube laps with the inlet velocity of $13 \mathrm{~m} / \mathrm{s}$ and gas void fraction of $90 \%$. The upper and the lower rows represent the first and the last sections of the hole, respectively, in each lap. The oriented lines in each section are the projection component of spatial streamline on each section. From the

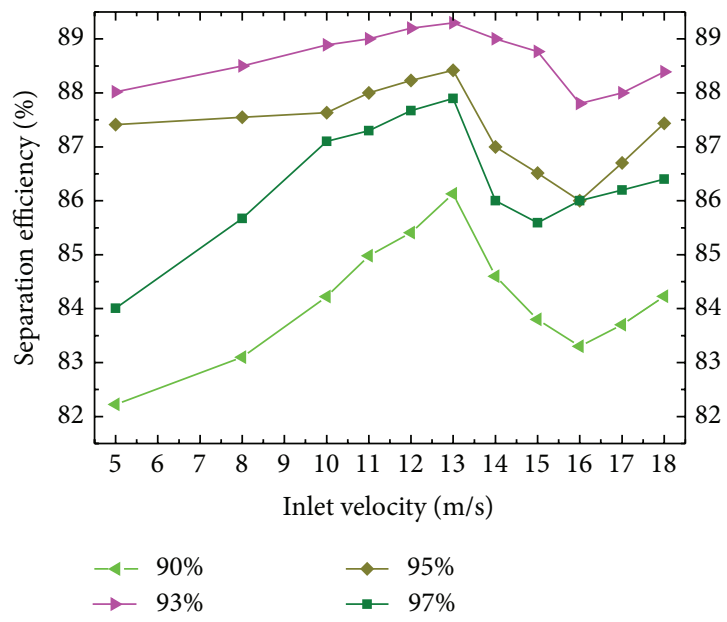

FIGURE 10: The influence of inlet velocity on separation efficiency.

contour of the gas void fraction it can be seen that the air gathers in the inner side of helically coiled tube and the water concentrates on outside under the combined actions of the gravity and the centrifugal forces. The separation effect has been strengthened after each helically coiled tube lap. In the first five laps the separation effect basically reaches the limit and there will be no significant improvement. Compare the 


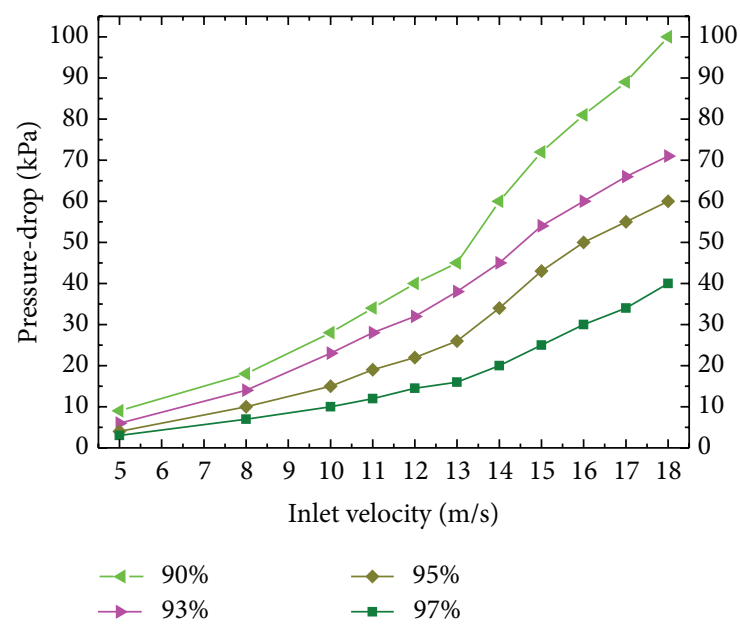

FIgURE 11: The influence of inlet velocity on pressure-drop.

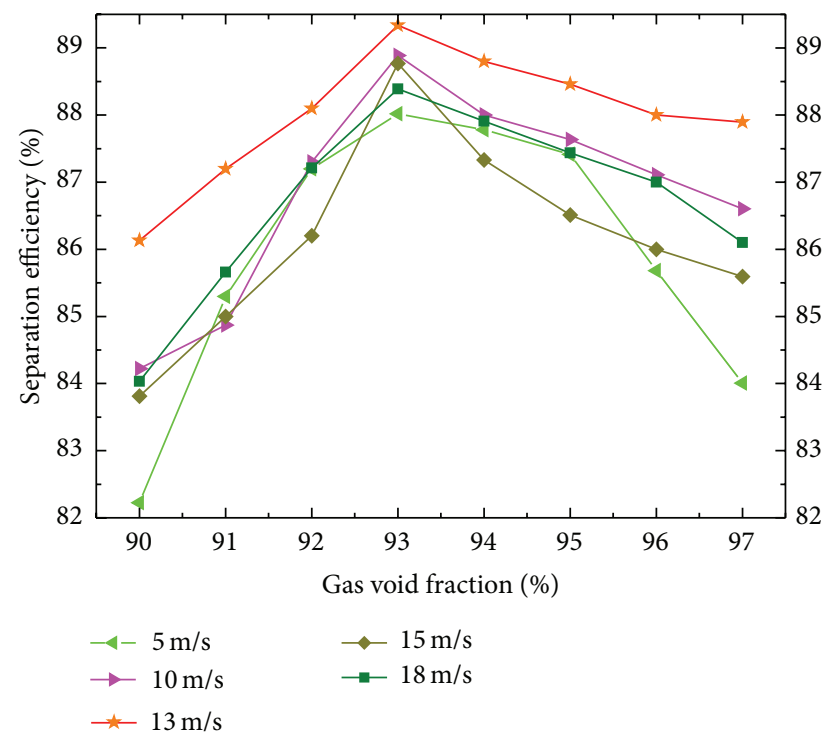

Figure 12: The influence of gas void fraction on separation efficiency.

first and the last hole section in each lap, it is obvious to find that each outflow of gas flow at holes disturbs the whole inner flow field of the helically coiled tube and weakens the liquid layered effect, leading to less outflow for high gas void fraction gas and this effect will be cumulative to the maximum for the last one outflow hole of each lap. The spacing between holes can be regarded as "adjustment segment." By increasing the spacing, the mixture of stratified flow with bad separation effect gets fully adjusted to deteriorate, to recover, and further to improve the separation efficiency [22].

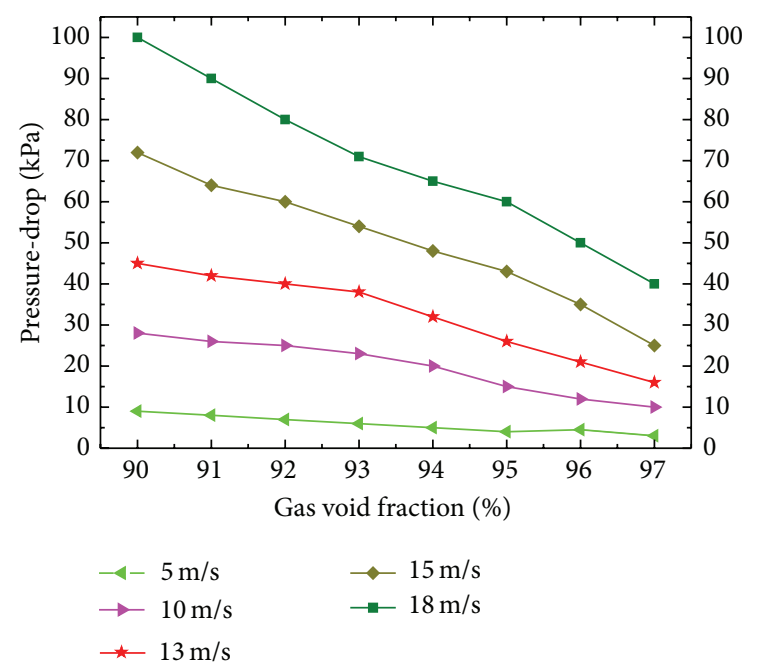

FIGURE 13: The influence of gas void fraction on pressure-drop.

\section{Comparison with the Experimental Data and Numerical Result}

In order to verify the reliability of the selected models, five conditions at inlet velocities of $5 \mathrm{~m} / \mathrm{s}, 10 \mathrm{~m} / \mathrm{s}, 13 \mathrm{~m} / \mathrm{s}, 15 \mathrm{~m} / \mathrm{s}$, and $18 \mathrm{~m} / \mathrm{s}$ are chosen to make comparison.

6.1. The Comparison of Separation Efficiency. The experimental and numerical separation efficiency of the helically coiled tube separator with gas void fraction are compared in Figure 15 under several inlet velocity points of $5 \mathrm{~m} / \mathrm{s}, 10 \mathrm{~m} / \mathrm{s}$, $13 \mathrm{~m} / \mathrm{s}, 15 \mathrm{~m} / \mathrm{s}$, and $18 \mathrm{~m} / \mathrm{s}$. From Figure 15 it can be known that the experimental data and numerical result have the same regularity trend, and the numerical value is lower. This is because the boundary conditions set in simulations are different with the actual experimental conditions, such as the droplet diameter of $0.1 \mathrm{~mm}$ and the bubble diameter of $1 \mathrm{~mm}$. However in the actual experiment, the two-phase particles are deformed at all times. What is more, the gas-liquid flow rate may not be very accurate during the experiment. The inlet and outlet of the separator are equipped with valves which influence the inlet velocity of gas-liquid two-phase and the degree of mixing. Meanwhile, the process parameters are also affected by air temperature, humidity, and other factors. These above all are the reasons leading to the difference of the separation efficiency.

6.2. The Comparison of Pressure-Drop. The experimental and numerical pressure-drop of the helically coiled tube with gas void fraction are compared in Figure 16 under several inlet velocity points of $5 \mathrm{~m} / \mathrm{s}, 10 \mathrm{~m} / \mathrm{s}, 13 \mathrm{~m} / \mathrm{s}, 15 \mathrm{~m} / \mathrm{s}$, and $18 \mathrm{~m} / \mathrm{s}$. From Figure 16 it can be seen that the experimental data and numerical result share the same trend, and the numerical value is higher. This is mainly because the simulations are performed in an ideal state that the holes at inner side of the helically coiled tube and the outlet of the tube are set at the same pressure condition while it is not the same as the experiment. In addition the separator entrance is connected 


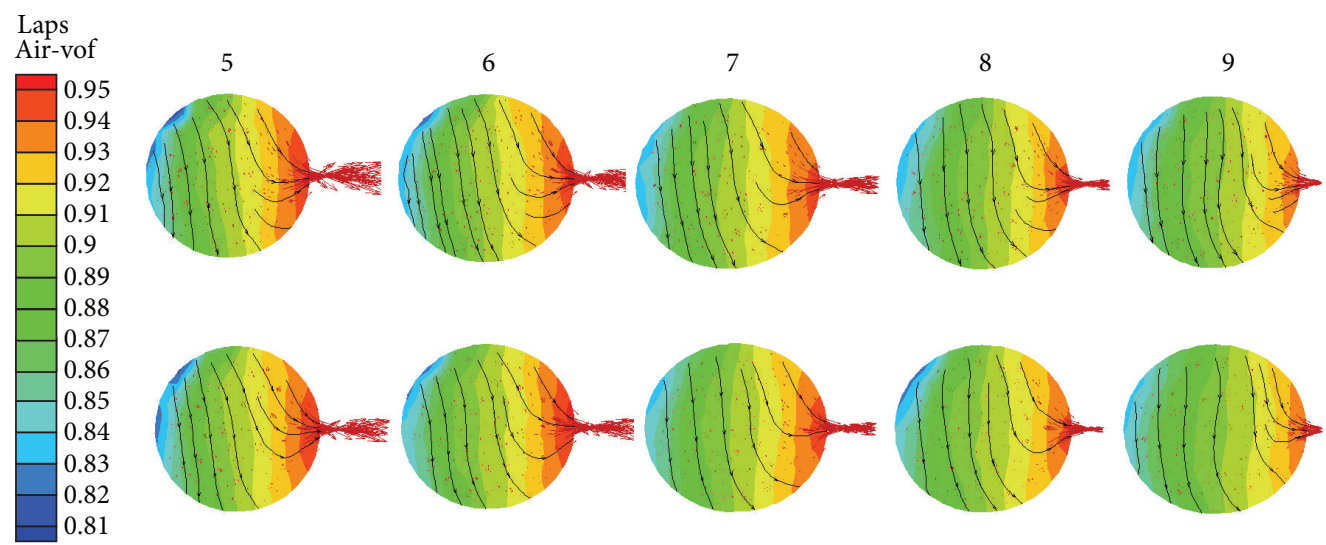

Figure 14: The flow field and contour of gas void fraction on different sections at laps.

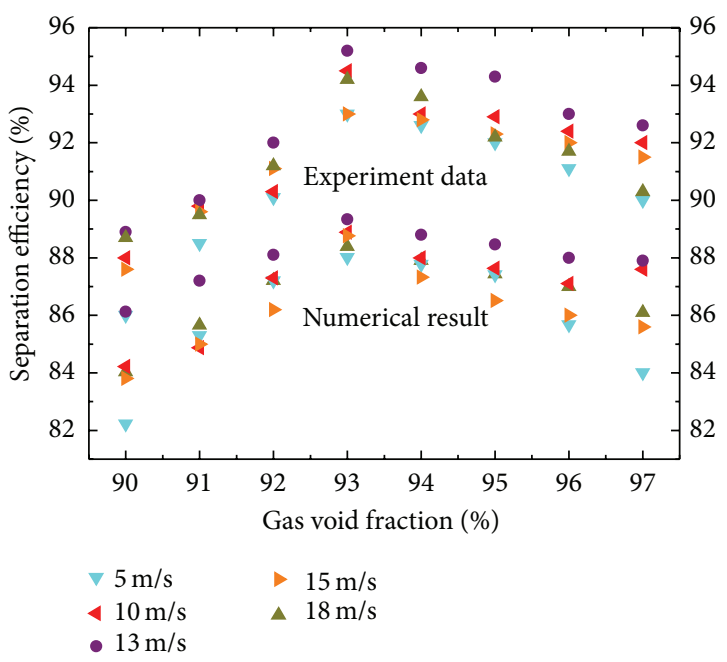

FIGURE 15: The comparison of separation efficiency between experiment data and numerical result under different velocities.

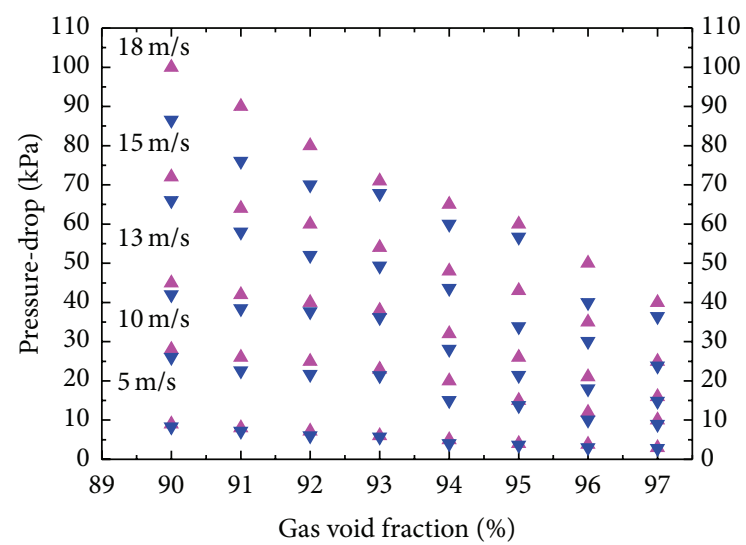

A Numerical result

$\checkmark$ Experiment data

FIGURE 16: The comparison of pressure-drop between experiment data and numerical result under different velocities. with a certain distance of pipeline and nozzle during the experiments which have led to the deviation between the experimental data and numerical result.

After larger numbers of data comparisons and verifications, the difference between numerical and experimental results is within the allowable range, and thus the numerical models of the helically coiled tube separator are reliable which can be used for more conditions in the future research.

\section{Conclusions}

(1) At a constant gas void fraction of the gas-liquid mixture, the gas-liquid separation efficiency increases with the inlet velocity in the range of 5 to $13 \mathrm{~m} / \mathrm{s}$. At an inlet velocity of $13 \mathrm{~m} / \mathrm{s}$, the separation efficiency reaches the peak value. If the inlet velocity further increases, the separation efficiency decreases first and increases again. Meanwhile, the pressure-drop shows a manner of quadratic behavior with the inlet velocity in the range of 5 to $18 \mathrm{~m} / \mathrm{s}$.

(2) At a constant inlet velocity of the gas-liquid mixture, the gas-liquid separation efficiency increases with the gas void fraction in the range of 88 to $97 \%$. At the gas void fraction of 93 to $94 \%$, the separation efficiency reaches the peak value. And with the gas void fraction further increasing, the separation efficiency decreases. Meanwhile the pressure-drop decreases with the inlet velocity increasing.

(3) From the comparison analysis of the separator efficiency and the pressure-drop between the numerical and experimental results it can be seen that both show the same regularity trend and the average deviation is within the allowable range of $11 \%$, which verifies the reliability of the numerical models. Above all, the optimal operating parameters of the helically coiled tube separator are inlet velocity of $13 \mathrm{~m} / \mathrm{s}$ and gas void fraction of $93 \%$ and the separation efficiency and pressure-drop are $95.2 \%$ and $0.3 \mathrm{MPa}$, respectively. 


\section{Conflict of Interests}

The authors declare that there is no conflict of interests regarding the publication of this paper.

\section{Acknowledgments}

This work was supported by the key cross-disciplinary of Low Carbon Energy Project and the Science Foundation of China University of Petroleum, Beijing (no. KYJJ2012-04-11). The authors would like to express their great thanks heartily.

\section{References}

[1] Y. F. Ma and Y. L. Li, "Analysis of the supply-demand status of China's natural gas to 2020," Petroleum Science, vol. 7, no. 1, pp. 132-135, 2010.

[2] C. Z. Zheng, Y. Zhou, Z. J. Guo et al., "Separation technique for oil-gas multi-phase flow transportation in submarine pipeline," in Proceedings of the 17th Water Dynamics Conference, pp. 830837, Hong Kong, 2004.

[3] Q. Yang, "Analysis of present situation of studying new gas recovery technique by downhole gas liquid separation abroad," Natural Gas Industry, vol. 21, no. 2, pp. 85-88, 2001.

[4] Y. Zhou, Y. X. Wu, Z. C. Zheng et al., "Research on oil-water separation technique I-numerical simulation in both straight and helical pipes," Journal of Hydrodynamics, vol. 19, no. 4, pp. 541-546, 2004.

[5] L. J. Guo, G. J. Li, X. J. Chen et al., "Flow patterns and their transition characteristics of gas-oil two-phase flow in horizontal helically coiled tube," Journal of Engineering Thermo Physics, vol. 11, no. 4, pp. 472-476, 1996.

[6] H. Gao, L. J. Guo, and X. M. Zhang, "Study of the particle phase distribution of liquid-solid two phase flow in a helically coiled tube separator," Journal of Engineering Thermo Physics, vol. 23, supplement 1, pp. 205-208, 2002.

[7] H. Gao, L. J. Guo, and X. M. Zhang, "Particle phase distribution in gas-liquid-solid three-phase flow through helically coiled tube," Journal of Engineering Thermo Physics, vol. 25, no. 1, pp. 69-72, 2004.

[8] D. T. Gong, Y. X. Wu, Z. C. Zheng et al., "Numerical simulation of the oil-water two-phase flow in a helical tube with variable mass flow rates," Journal of Hydro Dynamics Series A, vol. 21, no. 5, pp. 640-645, 2006.

[9] C. Y. Li, G. Y. Ma, B. C. Zhu, K. Luo, and H. Wang, "Numerical simulation of water-oil separation in spiral pipe," Chemical Engineering, vol. 39, no. 8, pp. 57-60, 2011.

[10] C. Y. Li, G. Y. Mu, and X. Liu, "Numerical simulation on internal flow field of spiral pipe," Journal of Liaoning Shihua University, vol. 31, no. 3, pp. 34-37, 2011.

[11] C. Y. Li, M. Y. G, X. Liu et al., "Numerical simulation on oilwater separation in spiral pipe after different flow velocity," Journal of Liaoning Shihua University, vol. 32, no. 1, pp. 32-35, 2012.

[12] T. Frising, D. Thomas, D. Bémer, and P. Contal, "Clogging of fibrous filters by liquid aerosol particles: experimental and phenomenological modelling study," Chemical Engineering Science, vol. 60, no. 10, pp. 2751-2762, 2005.

[13] B. S. Li, Z. L. Ji, and H. Y. Chen, "Experimental studies on gas/liquid separation performance of natural gas filters," Natural Gas Industry, vol. 27, no. 10, pp. 123-125, 2007.
[14] C. Guo, Experimental and numerical investigation on gas-liquid separation of spiral pipe separator [M.S. thesis], University of Petroleum, Beijing, China, 2013.

[15] G. J. Li, L. J. Guo, H. Gao et al., "Flow patterns of oil-water liquid-liquid two-phase flow in hellcally coiled tubes," Journal of Chemical Industry and Engineering, vol. 51, no. 2, p. 242, 2000.

[16] M. H. Jiang, W. Jiang, G. Y. Zhang et al., "Structural design and experiment study of the hydro-cyclone for fine particle," Journal of the Daqing Petroleum Institute, vol. 29, no. 1, pp. 58-60, 2005.

[17] Y. Zhou, Y. X. Wu, Z. C. Zheng et al., "Research on oil-water separation technique I-numerical simulation in both straight and helical pips," Science \& Technology A, vol. 19, no. 4, 2004.

[18] D. A. Drew and R. T. Lahey, Particulate Two-Phase Flow, Butterworth-Heinemann, Boston, Mass, USA, 1993.

[19] C. G. Speziale and S. Thangam, "Analysis of an RNG based turbulence model for separated flows," International Journal of Engineering Science, vol. 30, no. 2, pp. 1379-1388, 1992.

[20] J. P. van Doormaal and G. D. Raithby, "Enhancements of the SIMPLE Method for predicting incompressible fluid flows," Numerical heat transfer, vol. 7, no. 2, pp. 147-163, 1984.

[21] J. H. Ferziger and M. Peric, Computational Methods for Fluid Dynamics, Springer, Heidelberg, Germany, 1996.

[22] Z. B. Wang and Y. H. Jin, "Research on vane-guided cyclone for gas-liquid separation," Fluid Machinery, vol. 34, no. 3, pp. 7-10, 2006. 

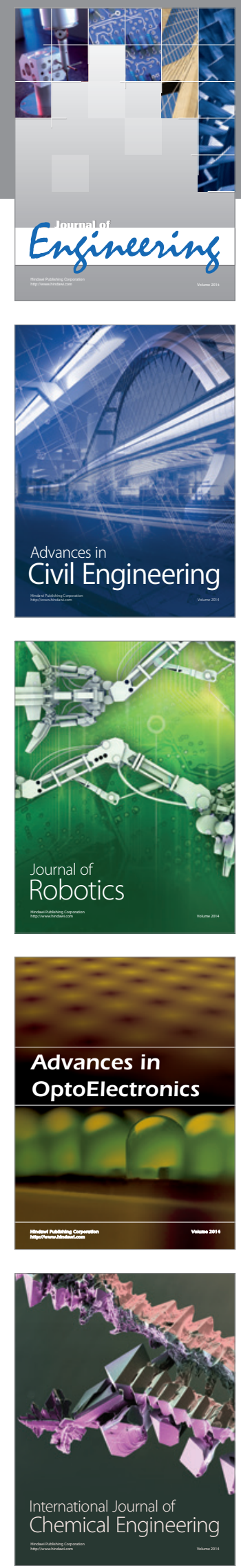

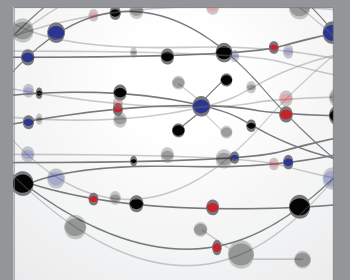

The Scientific World Journal
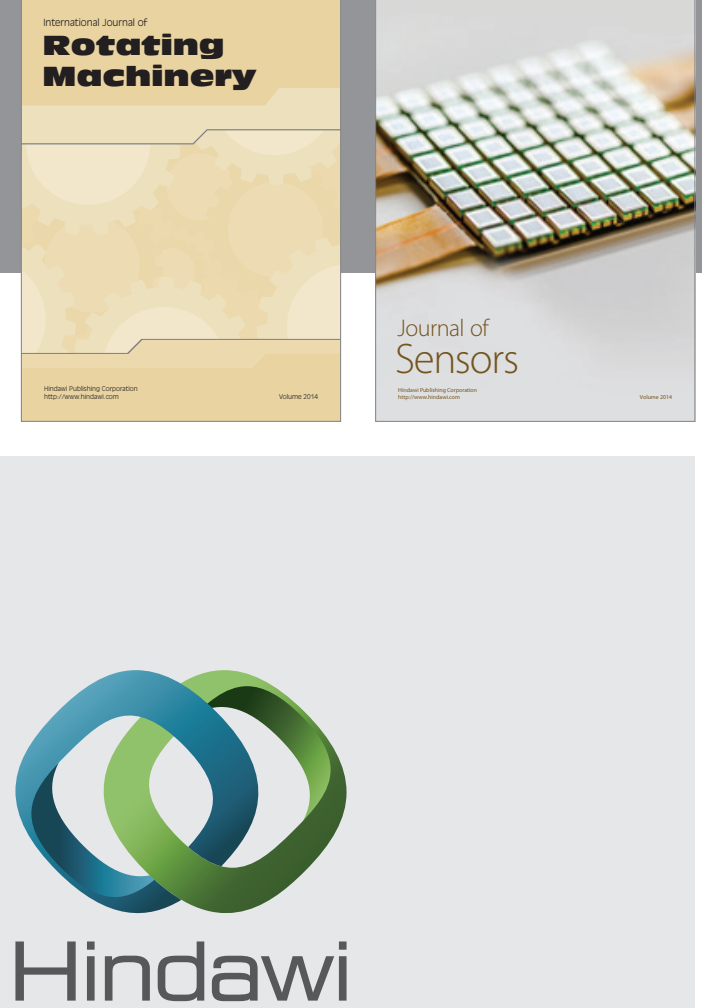

Submit your manuscripts at http://www.hindawi.com
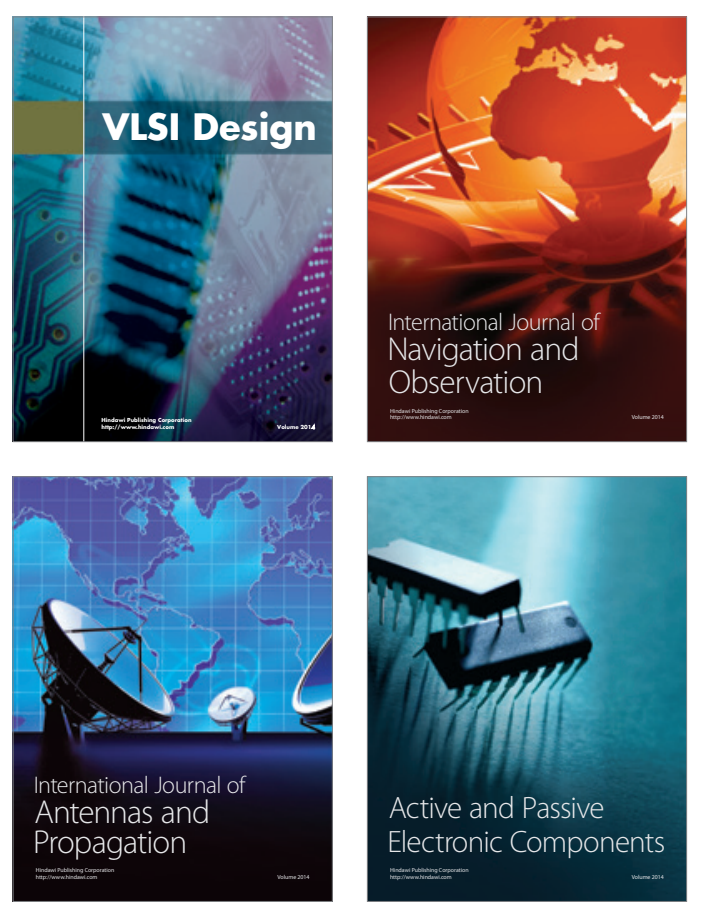
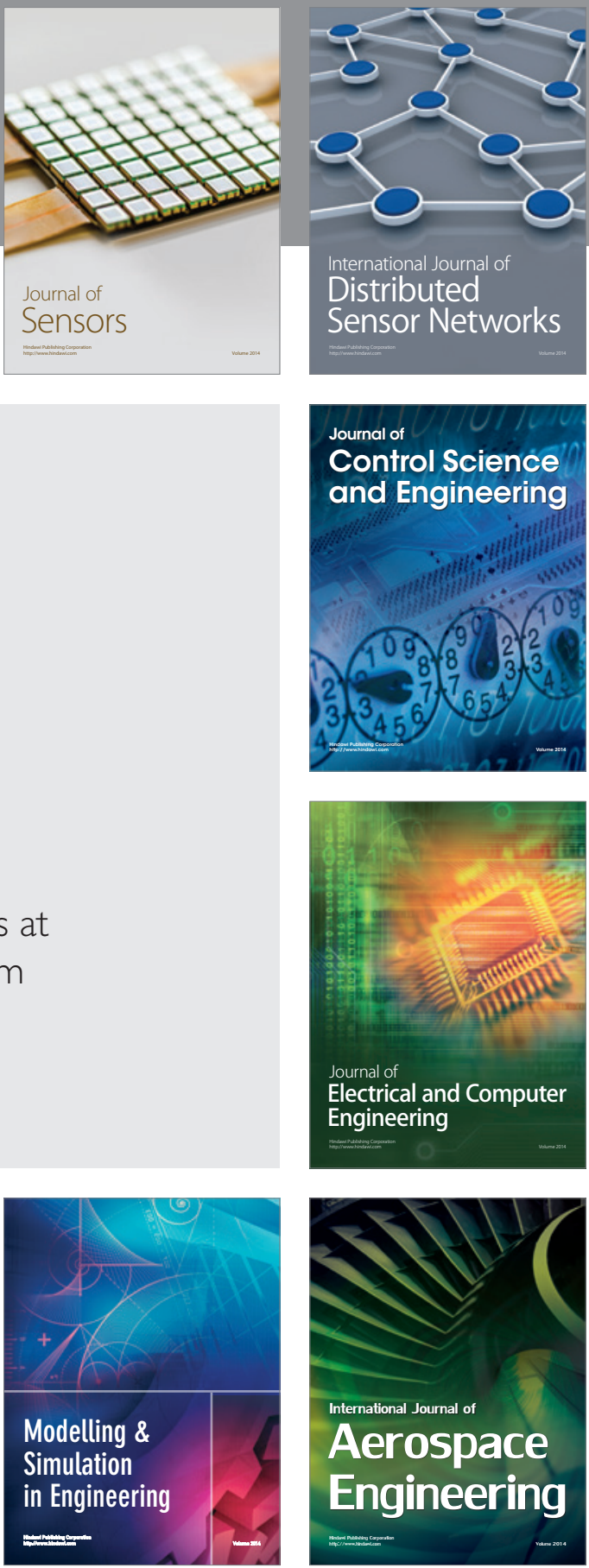

Journal of

Control Science

and Engineering
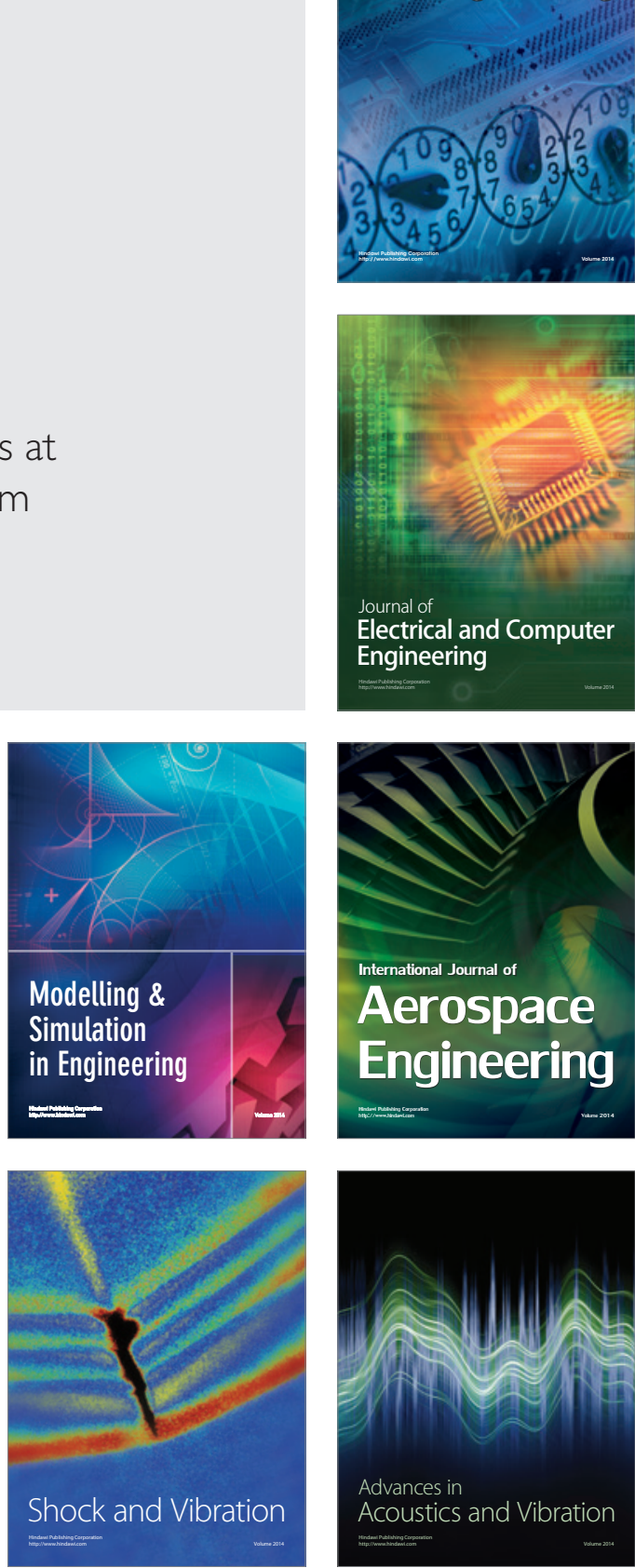